\title{
Análisis del tratamiento en medio ácido de partículas de pizarra
}

\author{
$M^{a}$. A. RODRÍGUEZ ${ }^{A}$ F. RUBIO ${ }^{B}$, J. RUBIOB , A. MURCIEGOA , J. L. OTEO \\ ${ }^{a}$ Area de Cristalografía y Mineralogía.. Universidad de Extremadura. Badajoz. \\ 'Instituto de Cerámica y Vidrio. (CSIC). Arganda del Rey. Madrid.
}

\begin{abstract}
En este trabajo se ha llevado a cabo el tratamiento de partículas de pizarra en medio ácido. Los resultados han sido analizados en función de los iones extraídos y de la variación que dicho tratamiento ocasiona en los valores de la superficie específica de las muestras estudiadas. Se han empleado también las técnicas de espectroscopía infrarroja (FT-IR) y difracción de rayos X. Se ha comprobado que la pizarra está compuesta fundamentalmente por cuarzo, moscovita y clorita, siendo ésta última extraída durante el ataque ácido. Los valores de la superficie específica disminuyen con el tratamiento ácido, lo que corrobora los resultados de las otras técnicas experimentales, es decir, la extracción de clorita de las partículas de pizarra.
\end{abstract}

Palabras Clave: pizarra, ataque ácido, clorita, moscovita, disolución, área superficial.

\section{Analysis of the aqueous acid attack of slate particles}

Slate particles were treated in liquid acid medium. The results were analyzed as a function of the disolved ions and specific surface area values. Infrared spectroscopy (FT-IR) and X- ray difraction were also used in this study. Slate particles are composed by quartz, muscovite and chlorite. Chlorite is the mainly phase leached during the acid attack. The specific surface area of the treated particles decreases with the intensity of the treatment, being this result assigned to the elimination of chlorite from the slate particles. FT-IR, X- ray diffraction and the analysis of the leached ions confirms this assumption.

Key Words: slate, acid attack, chlorite, moscovite, ion disolution, surface area.

\section{INTRODUCCIÓN}

El ataque en medio ácido de minerales arcillosos es un método muy empleado para modificar las propiedades físicoquímicas de dichos materiales. Generalmente el tratamiento en medio ácido ocasiona un aumento en los valores de superficie específica debido a diversos factores como son: la desaglomeración de las partículas existentes, la eliminación de impurezas y el intercambio iónico protón-catión metálico (1). Estos cambios superficiales son de gran importancia para las aplicaciones posteriores de dichos minerales: decolorantes, adsorbentes, catalizadores y soportes de catalizadores, portadores de pesticidas, etc. (2-4). Por lo que respecta a la pizarra, ésta ha sido muy poco utilizada en otras aplicaciones distintas a las de techado de edificios o plaquetas para cubierta de suelos y paredes. No obstante, gran parte de las pizarras extraídas de la cantera poseen defectos que las hacen inviables para las aplicaciones anteriormente mencionadas, por lo que son acumuladas en escombreras, ocasionando problemas de espacio, ambientales, etc. Es por ello necesario buscar nuevas aplicaciones para la pizarra, por ejemplo, su utilización como carga en resinas orgánicas para obtener materiales compuestos (5).

$\mathrm{Al}$ igual que ocurre con minerales arcillosos, las partículas de pizarra pueden ser activadas mediante ataque en medio ácido (6). El tratamiento en medio ácido de dichos minerales suele denominarse "activación ácida", ya que generalmente produce un aumento de la superficie específica y del número de centros activos en el material. La "activación ácida" consiste, esencialmente, en el tratamiento con ácidos tales como
$\mathrm{H}_{2} \mathrm{SO}_{4^{\prime}} \mathrm{HNO}_{3^{\prime}} \mathrm{y}$, especialmente, el $\mathrm{HCl}$. La intensidad del tratamiento dependerá de factores tales como la naturaleza y concentración del ácido utilizado, el tiempo de tratamiento y la temperatura. Son muchos los trabajos que han demostrado este tipo de activación (1, 4, 7-9).

Para estudiar la "activación ácida" de distintos minerales cristalinos o vítreos, se han utilizado diferentes técnicas: espectroscopía infrarroja $(1,10)$, microscopía electrónica de transmisión $(4,7)$, difracción de rayos X (9) y análisis de los iones extraídos $(9,10)$. La cantidad de iones eliminados durante el tratamiento en medio ácido depende de la composición química y de la estructura cristalina del material, así como de la intensidad del ataque. Se ha comprobado que si el tratamiento es agresivo, los cationes octaédricos son fácilmente disueltos, mientras que los que ocupan posiciones tetraédricas llegan a formar capas de sílice amorfa, insolubles en la disolución ácida (1). Estas capas son las responsables del aumento de porosidad y de superficie específica normalmente observados, así como del incremento de centros activos en la superficie del material.

En este trabajo se estudia el ataque en medio ácido de partículas de pizarra, con el propósito de aumentar el número de centros activos y así lograr una mayor interacción con las matrices orgánicas cuando sean preparados los materiales compuestos. Los estudios han sido seguidos mediante espectroscopía infrarroja, difracción de rayos $\mathrm{X}$, determinación de la superficie específica y análisis de los iones disueltos tras el ataque. 


\section{EXPERIMENTAL}

Las partículas de pizarra utilizadas en este trabajo fueron obtenidas a partir de planchas de $10 \times 10 \times 0,5 \mathrm{~cm}$ suministradas por Pizarras de Villar del Rey, Badajoz (España). Estas planchas fueron molturadas en molino de bolas durante 10 minutos, primero, y después en molino de ágata, recogiéndose la fracción inferior a $53 \mu \mathrm{m}$. La composicion química y mineralógica ha sido dada en otros trabajos $(11,12)$, siendo mayoritariamente: $\mathrm{SiO}_{2}(59 \%), \mathrm{Fe}_{2} \mathrm{O}_{3}(7 \%), \mathrm{TiO}_{2}(2 \%), \mathrm{Al}_{2} \mathrm{O}_{3}(21 \%), \mathrm{MgO}$ $(2 \%), \mathrm{K}_{2} \mathrm{O}(2 \%)$, y otros componentes minoritarios como $\mathrm{CaO}$ $(0,3 \%), \mathrm{Na}_{2} \mathrm{O}(0,5 \%)$ y $\mathrm{SO}_{3}(0,2 \%)$, y minerales de moscovita (sericita), clorita, cuarzo y feldespatos.

Los ataques en medio ácido fueron realizados en un reactor de teflón, homogeneizando la muestra y la solución ácida mediante un agitador de palas a una velocidad de 1.200 r.p.m., con el fin de mantener las partículas en constante suspensión. El reactor se termostatizó exteriormente mediante una camisa por la que circulaba, de forma continua, agua a temperatura de $50^{\circ} \mathrm{C}\left( \pm 0,5^{\circ} \mathrm{C}\right)$. Después de 1 hora se añadieron las partículas de pizarra y se mantuvieron en la disolución ácida durante tiempos de 2, 5, 10, 30, 90 minutos y 24 horas. La disolución atacante se preparó diluyendo en agua desionizada los volúmenes de $\mathrm{HCl}$ concentrado necesarios para obtener valores de $\mathrm{pH} 1,2$ y 3.

Una vez terminada la reacción de ataque en medio ácido, las partículas de pizarra se filtraron mediante un sistema milipore con recipientes de plástico y filtros de celulosa de tamaño de poro inferior a $0,5 \mu \mathrm{m}$, conectados a una bomba de vacío de membrana para acelerar la filtración. Las partículas de pizarra se lavaron sucesivas veces y posteriormente se secaron en estufa a $50^{\circ} \mathrm{C}$, guardándose en un desecador para su posterior análisis. La solución filtrante también se recogió para el análisis de los iones extraídos.

Las partículas de pizarra atacadas fueron analizadas mediante difracción de rayos $X$, utilizándose un difractómetro de rayos X Siemens D-500, y radiación Cu-K $K_{\alpha}(\lambda=1,54050 \AA$ A $)$ y mediante espectroscopía infrarroja, empleándose un espectrofotómetro infrarrojo por transformadas de Fourier (FT-IR) Perkin-Elmer, mod. 1760X, de $2 \mathrm{~cm}^{-1}$ de resolución. Se realizaron 10 espectros entre 4.000 y $400 \mathrm{~cm}^{-1}$, a los que se restó, en todos los casos, el ruido de fondo. Para preparar la pastilla a analizar se utilizó $1 \mathrm{mg}$ de muestra y 300 mg de KBr prensándose hasta 8 toneladas durante 2 minutos. La medida de la superficie específica se realizó en un equipo construido en el laboratorio y basado en el método cromatográfico de NelsenEggertsen (13), en el cual se utiliza una corriente de $\mathrm{He} / \mathrm{N}_{2}$ a presión parcial de 0,35 . La adsorción de nitrógeno se realiza a la temperatura de $77 \mathrm{~K}$, mientras que la desorción se realiza a temperatura ambiente (14). Las muestras fueron previamente desgasificadas a la temperatura de $120^{\circ} \mathrm{C}$ durante 18 horas.

Los iones existentes en las soluciones fueron analizados mediante un equipo ICP (plasma de acoplamiento inductivo) Jobin-Ybon, excepto el Na y K que se analizaron por fotometría de llama.

\section{RESULTADOS Y DISCUSIÓN}

En la Figura 1 se muestran los espectros FT-IR de las muestras atacadas en medio ácido a diferentes tiempos y $\mathrm{pH}$. En todos los espectros se pueden apreciar diferentes bandas atribuidas a los componentes de la pizarra. Así, en la zona de las vibraciones $\mathrm{OH}\left(4000-3000 \mathrm{~cm}^{-1}\right)$ aparecen los picos atribuidos

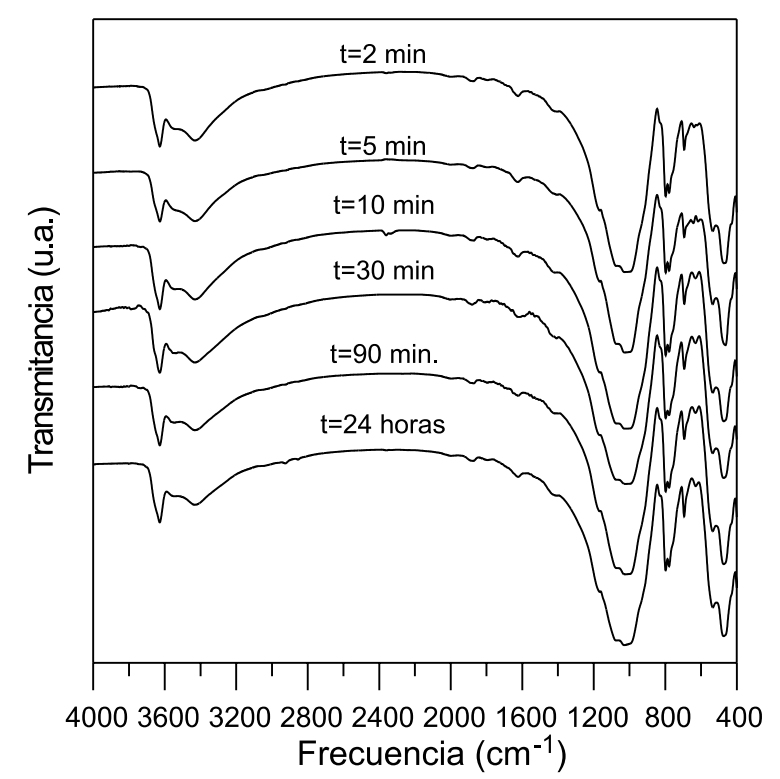

Figura 1.- Espectros infrarrojos de las partículas de pizarra atacadas a $\mathrm{pH}=\mathrm{l}$ y diferentes tiempos de reacción.

a la moscovita (situados a 3651 y $3525 \mathrm{~cm}^{-1}$ ) y los atribuidos la clorita (situados a 3560 y $\left.3430 \mathrm{~cm}^{-1}\right)(11,15)$. Por otro lado, las bandas que aparecen a $1165,696,530$ y $470 \mathrm{~cm}^{-1}$, así como el fuerte doblete situado a $798-780 \mathrm{~cm}^{-1}$ son indicativas de la presencia de cuarzo (15). El cuarzo también tiene dos bandas debidas a la tensión Si-O-Si situadas a 1150 y $1084 \mathrm{~cm}^{-1}$, las cuales están solapadas con las bandas correspondientes a las vibraciones de tensión de la red de la moscovita y de la clorita. La moscovita origina bandas fácilmente localizables a 1068, 1029 y $630 \mathrm{~cm}^{-1}$, mientras que la clorita origina bandas situadas a $1000,833,752$ y $471 \mathrm{~cm}^{-1}$, todas ellas solapadas con las de la moscovita (15), excepto el pequeño hombro que aparece a 660 $\mathrm{cm}^{-1}$, característico de la clorita, y que confirma la presencia de este mineral en la pizarra.

En la región espectral 1500-400 $\mathrm{cm}^{-1}$, los espectros FT-IR son prácticamente similares, con independencia del $\mathrm{pH}$ y del tiempo de ataque empleados. El único efecto observable es el cambio de transmitancia producido en la zona $900-600 \mathrm{~cm}^{-1}$, siendo más intensa la zona de $900 \mathrm{~cm}^{-1}$ que la de $600 \mathrm{~cm}^{-1}$ en la muestra sin atacar, mientras que en las muestras atacadas esta relación de intensidades se invierte (la zona de $600 \mathrm{~cm}^{-1}$ es ahora más intensa que la de $900 \mathrm{~cm}^{-1}$ ) a medida que el ataque es más largo.

En la región espectral 4000-3000 $\mathrm{cm}^{-1}$, se aprecian los cambios más importantes. Así, la banda atribuida a la moscovita $\left(3651 \mathrm{~cm}^{-1}\right)$ se hace bastante más intensa, o bien se diferencia más, de las bandas originadas por la clorita $\left(3560\right.$ y $\left.3430 \mathrm{~cm}^{-1}\right)$, lo cual indica que, o bien se están activando los hidroxilos de la moscovita, o bien es la clorita la que está siendo eliminada (extraída) de las partículas de pizarra durante el ataque ácido. A medida que el ataque es más intenso, estos efectos se hacen más acusados, tal y como puede verse en las Figuras 1 y 2.

La banda de vibración de flexión del grupo $\mathrm{OH}$ en agua molecular situada $1636 \mathrm{~cm}^{-1}$ es prácticamente similar en todos los espectros, lo que indica que las muestras atacadas no retienen agua tras el ataque.

En la Figura 3 se muestran los difractogramas de rayos $X$ de las partículas de pizarra sin atacar y atacadas en medio ácido. Los picos de difracción están bien definidos y corresponden a 
los minerales cuarzo, moscovita y clorita, fundamentalmente, con muy pequeñas cantidades de feldespatos $(5,11)$. En estos difractogramas se observa cómo los picos correspondientes a la clorita disminuyen muy ligeramente en intensidad, en comparación con los de cuarzo y moscovita, cuando el ataque es más intenso ( $\mathrm{pH}=1$ y 24 horas). Este resultado indica que posiblemente la clorita es eliminada durante el ataque, lo que confirma el aumento relativo de la intensidad de los picos infrarrojos de la moscovita, comentado anteriormente.

En las Figuras 4-10 se muestra la variación de los iones extraídos $\left(\mathrm{SiO}_{2}, \mathrm{Fe}_{2} \mathrm{O}_{3}, \mathrm{Al}_{2} \mathrm{O}_{3}, \mathrm{MgO}, \mathrm{CaO}, \mathrm{K}_{2} \mathrm{O}\right.$ y Na $\left.\mathrm{Na}_{2} \mathrm{O}\right)$ durante el ataque en función del tiempo y para distintos valores de $\mathrm{pH}$. La escasa extracción de $\mathrm{TiO}_{2}$ a $\mathrm{pH}=1$ (oscilando entre 0,02 y

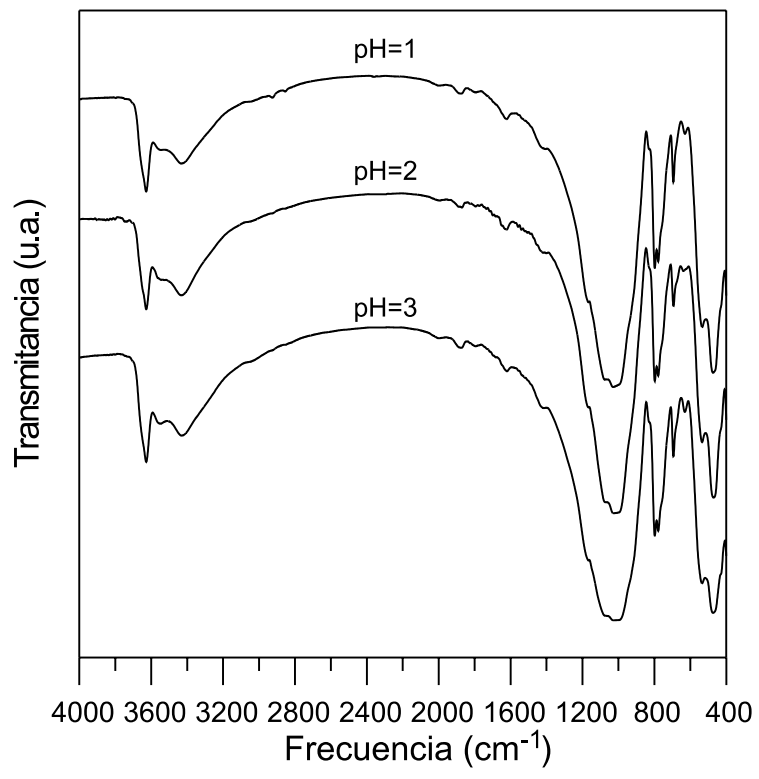

Figura 2.- Espectros infrarrojos de las partículas de pizarra atacadas al mayor tiempo y a diferentes $\mathrm{pHs}$.

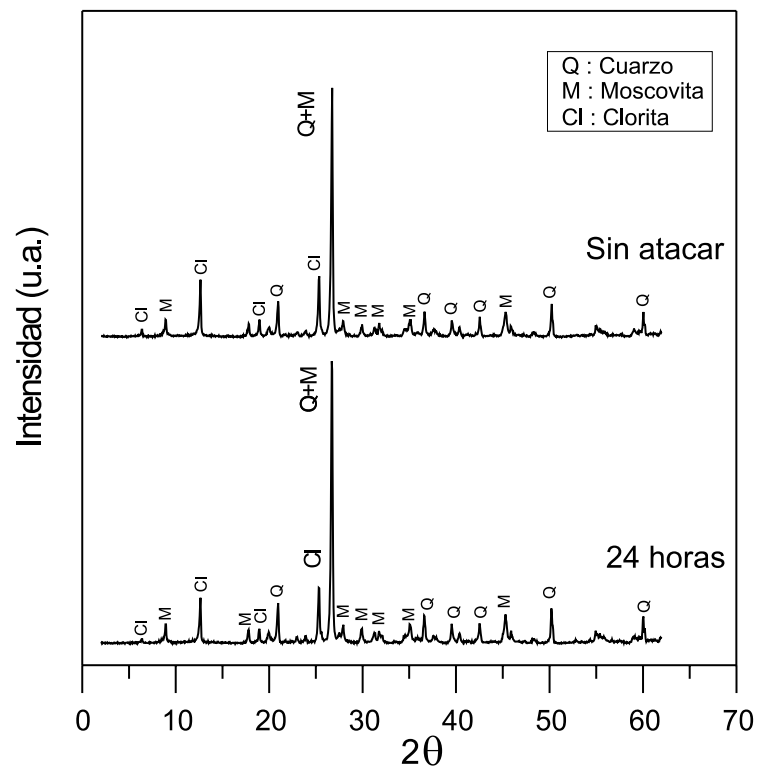

Figura 3.- Difractogramas de rayos $\mathrm{X}$ de las partículas de pizarra sin atacar y atacadas 24 horas a $\mathrm{pH}=1$.
0,08 para el menor y el mayor tiempo de ataque respectivamente), y nula para $\mathrm{pH}=2$ y $\mathrm{pH}=3$ nos lleva a no mostrar la figura correspondiente a la extracción de este catión.

A la vista de los resultados de las Figuras 4-10 deben considerarse varios aspectos . En primer lugar, puede comprobarse cómo para todos los cationes la cantidad extraída aumenta con la acidez del medio. Por lo que respecta al tiempo de ataque pueden observarse dos comportamientos; el primero, es el que corresponde a los elementos $\mathrm{Si}, \mathrm{Fe}, \mathrm{Al}$ y $\mathrm{Mg}$, los cuales se extraen más a medida que aumenta el tiempo de ataque, mientras que para los cationes $\mathrm{Ca}, \mathrm{K}$ y Na, la extracción aumenta en los primeros momentos para permanecer prácticamente estable para tiempos largos.

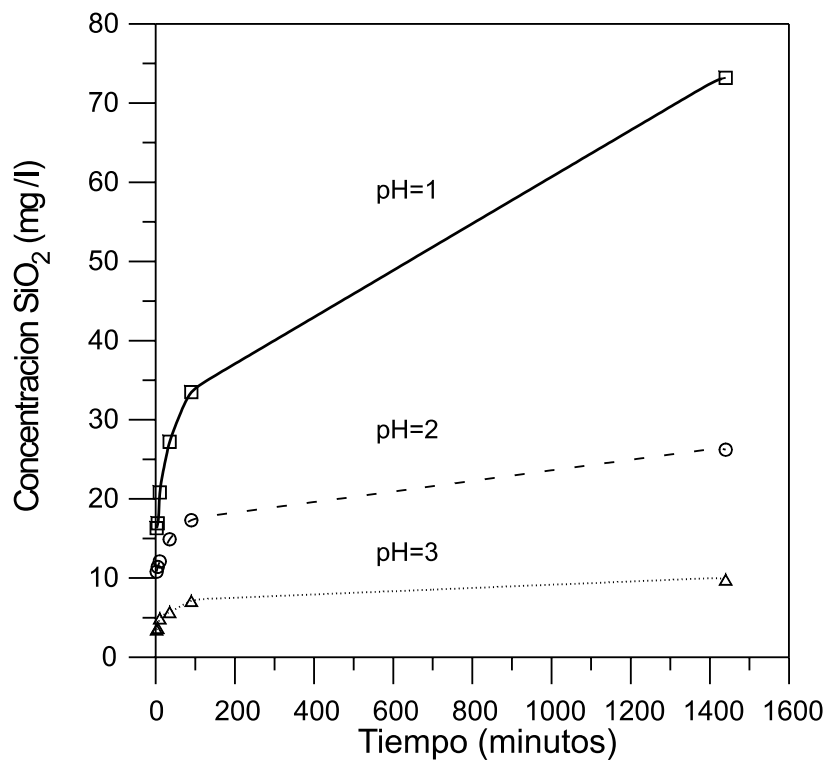

Figura 4.- Extracción de $\mathrm{SiO}_{2}$ de las partículas de pizarra en medio acuoso ácido.

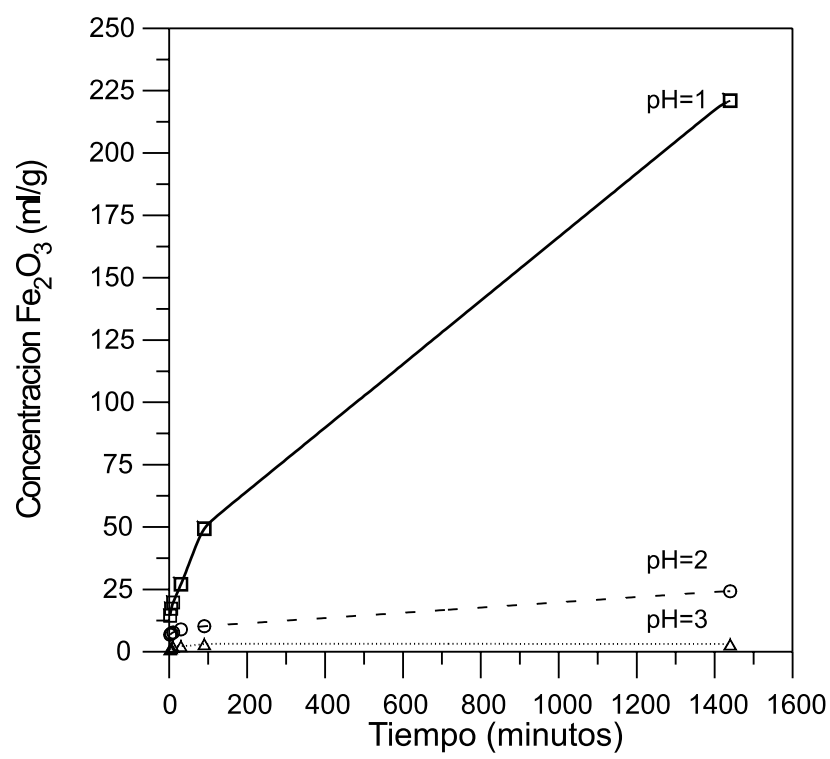

Figura 5.- Extracción de $\mathrm{Fe}_{2} \mathrm{O}_{3}$ de las partículas de pizarra en medio acuoso ácido. 


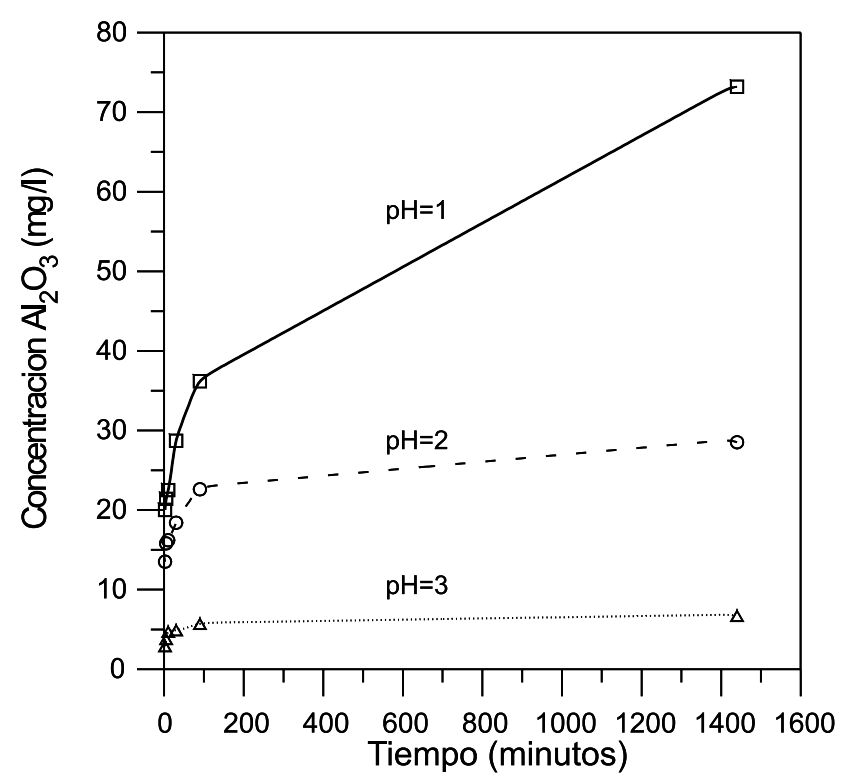

Figura 6.- Extracción de $\mathrm{Al}_{2} \mathrm{O}_{3}$ de las partículas de pizarra en medio acuoso ácido.

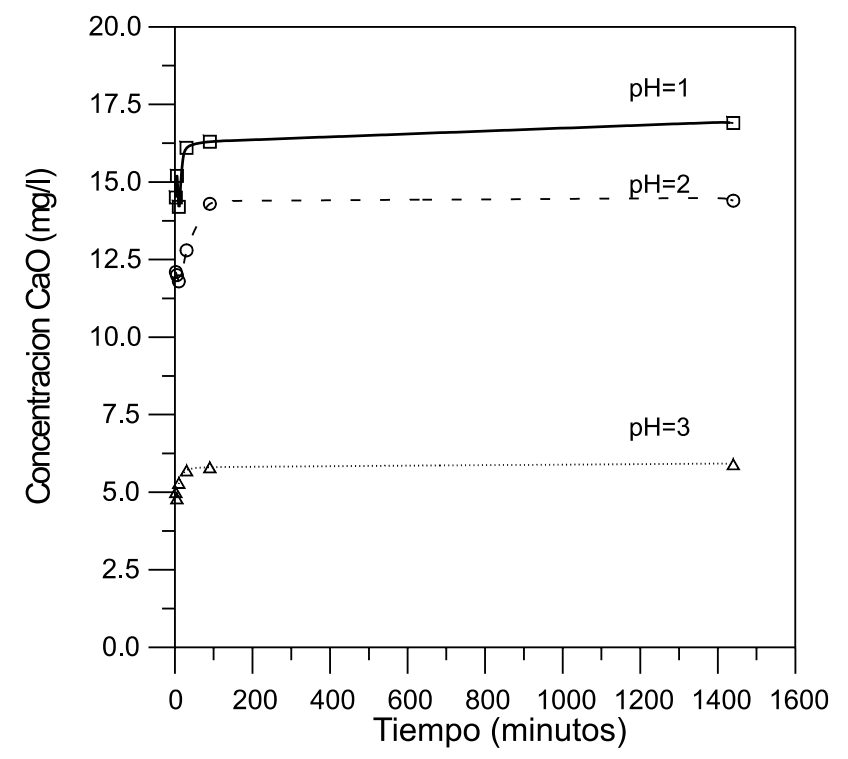

Figura 8.- Extracción de $\mathrm{CaO}$ de las partículas de pizarra en medio acuoso ácido.

La mayor extracción se produce en el Fe, llegándose a eliminar más de $200 \mathrm{mg} / 1$ a pH=1 y 24 horas de ataque, a pesar de que la concentración de este elemento en la pizarra es solamente del 7\%. Por otro lado la extracción de Si y Al es prácticamente similar para $\mathrm{pH}$ y tiempos iguales. Este resultado es debido a dos fenómenos, primero, la presencia de cuarzo en la pizarra y, segundo, a la mayor resistencia al ataque en medio ácido de la sílice frente a la alúmina $(5,10)$. La extracción de Mg es también bastante elevada si comparamos su concentración ( $2 \%)$ en la muestra de pizarra con la del K ( $2 \%)$, el cual se extrae en una proporción mucho menor.

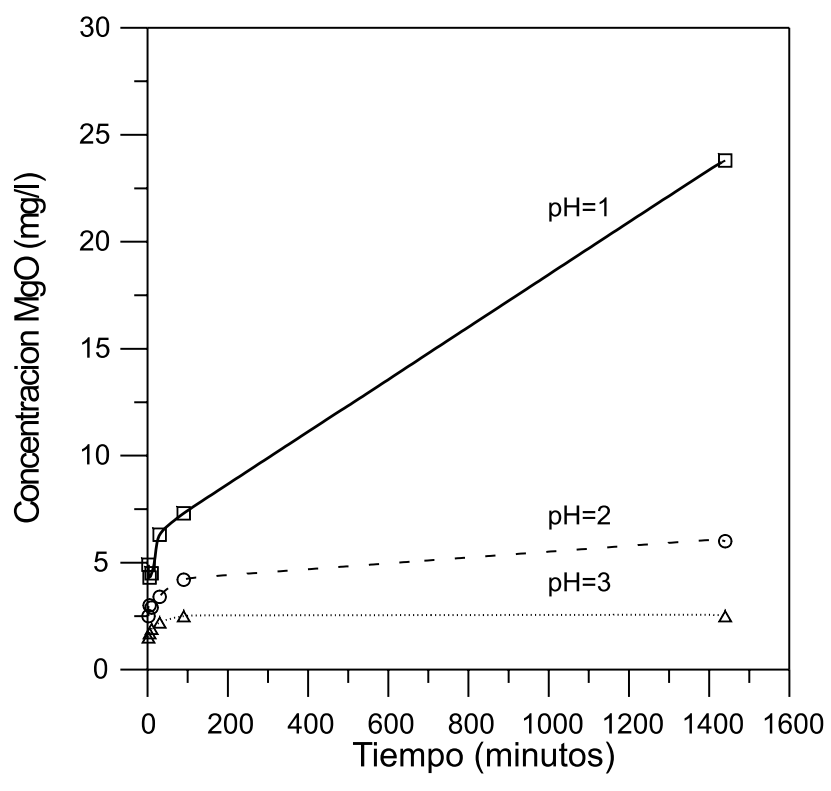

Figura 7.- Extracción de $\mathrm{MgO}$ de las partículas de pizarra en medio acuoso ácido.

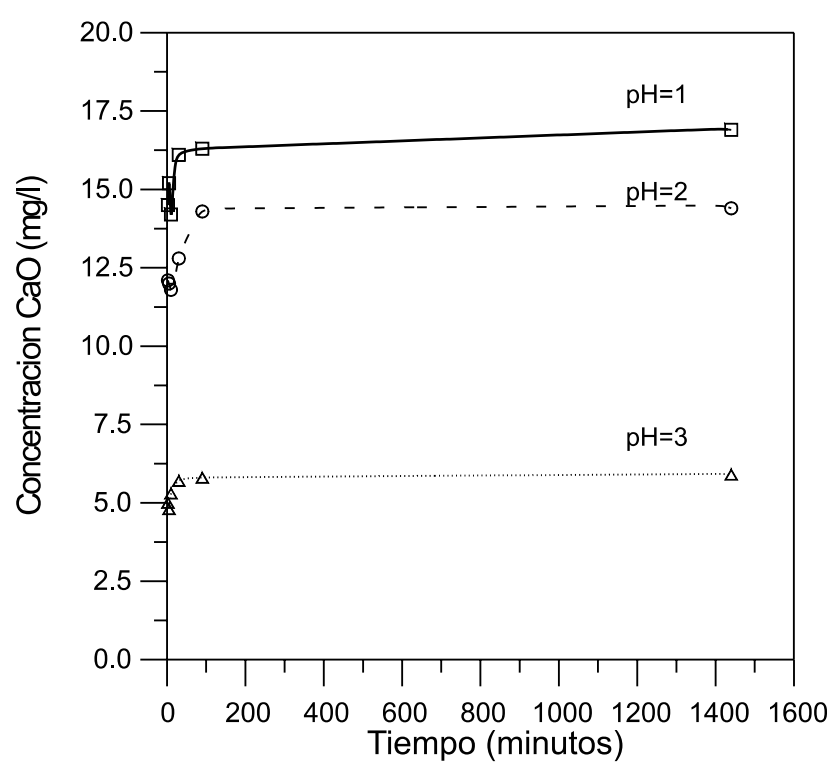

Figura 9.- Extracción de $\mathrm{K}_{2} \mathrm{O}$ de partículas de pizarra en medio acuoso ácido.

Si se tienen en cuenta las composiciones químicas de los tres minerales fundamentales, cuarzo, moscovita y clorita, existentes en la pizarra, pueden deducirse conclusiones importantes. Como es bien sabido, la fórmula química del cuarzo es $\mathrm{SiO}_{2}$, la de la moscovita puede representarse como $\mathrm{KAl}_{2}\left(\mathrm{AlSi}_{3} \mathrm{O}_{10}\right)(\mathrm{OH})_{2}$, y la de la clorita como $(\mathrm{Mg}, \mathrm{Fe})_{3}(\mathrm{Si}$, $\mathrm{Al})_{4} \mathrm{O}_{10}(\mathrm{OH})_{2}(\mathrm{Mg}, \mathrm{Fe})_{3}(\mathrm{OH})_{6}$. La presencia de $\mathrm{Na}$, Ca y otros elementos en la pizarra es debida a los feldespatos y a impurezas existentes en ella. Según estas formulaciones, el Fe y $\mathrm{Mg}$ proceden únicamente de la clorita, el $\mathrm{K}$ de la moscovita y el $\mathrm{Si}$ y $\mathrm{Al}$ pueden encontrarse en los tres minerales principales. 


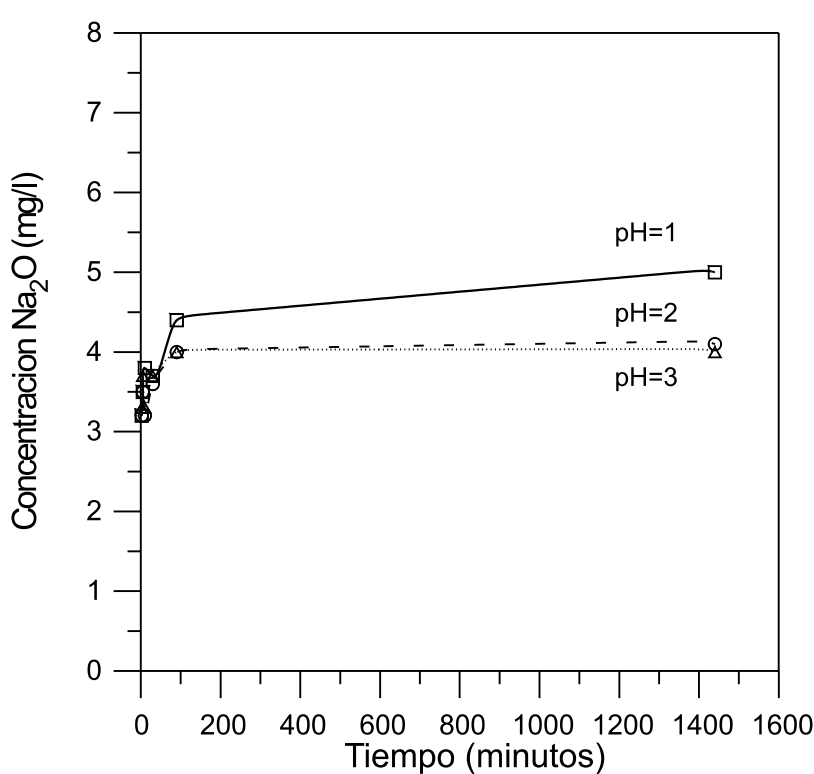

Figura 10. Extracción de $\mathrm{Na}_{2} \mathrm{O}$ de partículas de pizarra en medio acuoso ácido

TABLA I.- SUPERFICIE ESPECÍFICA DE LAS PARTíCULAS DE PIZARRA ATACADAS A DISTINTOS TIEMPOS.

\begin{tabular}{|c|cccccc|}
\hline $\begin{array}{c}\text { Tiempo de } \\
\text { reacción } \\
\text { (minutos) }\end{array}$ & 2 & 5 & 10 & 30 & 90 & 1440 \\
\hline $\mathrm{pH}=1$ & 5.54 & 5.53 & 5.43 & 5.30 & 5.19 & 4.12 \\
\hline $\mathrm{pH}=2$ & 5.73 & 5.69 & 5.50 & 5.36 & 5.29 & 4.95 \\
\hline $\mathrm{pH}=3$ & 5.80 & 5.79 & 5.71 & 5.61 & 5.56 & 5.38 \\
\hline
\end{tabular}

Comparando de nuevo los resultados de las Figuras 4-10, puede observarse cómo el Fe y Mg son extraídos en una cantidad bastante superior a los de los otros elementos, lo que indica que la clorita es más fácilmente atacable por las disoluciones ácidas. Este resultado confirma los ya expuestos mediante FT-IR y difracción de rayos X, en los que se apreciaba una disminución de las bandas o picos correspondientes a este mineral cuando las partículas de pizarra eran atacadas. Por lo tanto, el aumento observado en las bandas IR situadas a 3651 $\mathrm{cm}^{-1}$ (de la moscovita) con respecto a las otras bandas de la clorita es solamente un aumento relativo, pues lo que realmente sucede es una disminución de la cantidad de clorita en las partículas de pizarra, manteniéndose o disminuyendo muy ligeramente la proporción de moscovita.
Dado que en la clorita los iones Fe y Mg se encuentran en la capa octaédrica, los resultados obtenidos indican que ésta es la capa más fácilmente atacable, resultados similares a los ya encontrados por numerosos autores $(1,7,9,16,17)$.

Por otro lado, la extracción de Al, similar a la del Si, a pesar de su menor concentración, es debida a la eliminación de gran parte de la clorita y a una menor parte de moscovita. La extracción de moscovita es confirmada por el aumento de la cantidad de iones $\mathrm{K}$ disueltos, iones que solamente pueden provenir de la moscovita. Las curvas de extracción de $\mathrm{K}$ muestran que durante los primeros momentos de ataque es cuando se elimina la mayor cantidad de $K$, permaneciendo prácticamente constante la extracción entre 90 minutos y 24 horas. Este resultado indica que la moscovita es menos atacable que la clorita, lo que de nuevo confirma las conclusiones anteriores.

En la Tabla 1 se dan los valores de la superficie específica obtenidos para cada una de las muestras atacadas. Los resultados muestran claramente que todos los valores de la superficie específica son inferiores a los de las partículas de pizarra sin atacar $\left(5,8 \mathrm{~m}^{2} / \mathrm{g}\right)$, excepto para la muestra tratada a $\mathrm{pH}=3$ y 2 minutos, la cual presenta el mismo valor. También puede comprobarse cómo la disminución de la superficie específica es función del $\mathrm{pH}$ y del tiempo de ataque, siendo dicha disminución mayor a medida que el $\mathrm{pH}$ es más ácido y el tiempo más largo. Estos resultados muestran un comportamiento diferente al de otros minerales arcillosos, en los cuales la superficie específica aumenta a medida que el ataque se hace más intenso $(7,8,18,19)$. En éstos minerales el aumento se atribuye a la eliminación de los iones presentes en las estructuras octaédricas y la formación de una capa de sílice amorfa. Por otro lado, Suárez y col. (7) han encontrado que si la muestra posee mica y feldespatos, el aumento de la superficie específica es mucho menor o incluso puede disminuir, lo que es debido a que la mica y los feldespatos poseen valores de superficie específica muy bajos. Dado que la clorita posee mayor superficie específica que la moscovita y mucho mayor que el cuarzo y feldespatos existentes en las partículas de pizarra, el hecho de que las pizarras atacadas posean menores valores de superficie específica que la muestra sin atacar se debe a que se está eliminando, principalmente, clorita, quedando en dichas partículas una mayor proporción de cuarzo y moscovita. Este resultado corrobora los obtenidos mediante las otras medidas realizadas con FT-IR, DRX y análisis químico de los iones extraídos.

\section{CONCLUSIONES}

Los resultados obtenidos en este estudio han demostrado que las partículas de pizarra pueden ser "activadas" por tratamiento con disoluciones ácidas. La "activación" produce una eliminación de la clorita existente en dichas partículas, quedando en mayor proporción la moscovita. Esta "activación" es considerada desde el punto de vista de los hidroxilos libres presentes en la muestra, pues los hidroxilos de la moscovita poseen mayores frecuencias de vibración que los de la clorita, y por lo tanto, pueden considerarse más "libres" para interaccionar con otros compuestos. Sin embargo, la superficie específica disminuye durante la "activación", lo que es debido a la extracción de la clorita, la cual posee mayor superficie específica que el cuarzo o la moscovita existentes en las partículas de pizarra. La extracción de clorita se ha puesto de manifiesto mediante difracción de rayos $\mathrm{X}$ y, sobre todo, analizando los iones eliminados en la disolución atacante. 


\section{BIBLIOGRAFIA}

1. M.A. Vicente-Rodríguez, M. Suárez, M.A. Bañares-Muñoz, J.D. López-Gonzales. "Comparative FT-IR study of the rernoval of octahedral of naturaal cations and structural modifications during acid treatment of several silicates". Spectrochimica Acta. A. 52 (1996) 1685-1694.

2. J.M. Campelo, A. García, D. Luna, J.M. Marinas. "Catalytic activity of natural sepiolites in cyclohexene isomerization". Clay Miner. 22 (1987) 233 -236.

3. A. Corma, A. Mifsud, E. Sanz. "Catalytic activity of modified silicates: I. Dehydration of ethanol catalyzed by acidic sepiolite". Clay Miner. 22 (1987) 197205.

4. M. Myriarn, M. Suárez, J.M. Martín-Pozas. "Structural and textural modifications of palygorskite and sepiolite under acid treatment". Clays Clay Miner. 46 (1998) 3, 225-231.

5. $\mathrm{M}^{\mathrm{a}} \mathrm{A}$. Rodríguez, "Aprovechamiento de residuos de pizarra para su utilización en materiales compuestos". Tesis Doc. U. Extremadura. (1995).

6. MaA. Rodríguez, F. Rubio, J. Rubio, J L. Oteo. "Influence of the surface treatment of slate particles on their surface properties". To be published in Clay Clays Miner.

7. M. Suárez, L.V, Flores, M.A. Vicente, J.M. Martín-Pozas. "Acid activation of a palygorskite with HCI: Development of physico-chemical, textural and surface properties". Appl. Clay Sci. 10 (1995) 247-258.

8. M. Suárez, M.A. Vicente, R. Trujillano, C. Santiago, J.M. Martín-Pozas. "Acid activation of saponite from Cerro del Aguila (Madrid, Spain). Proc. $11^{\text {th }}$ International Clay Conf. (in Litt). Pp, 181- 184.

9. M. Suárez, L.V. Flores, J. Navarrete, A. Murciego, J.M. Martín-Pozas. "Estudio del tratamiento ácido de palygorskita". Actas del III Cong. Geológico Esp. y VIII Cong. Latinoamericano de Geología. Salamanca. Vol. 3. (1992) Pp. 347-3 51.
10. M.A. Rodríguez. Contribución al estudio del ataque de vidrios silicobóricos por disoluciones acuosas ácidas. Tesis Doc. U. Complutense. Madrid. (1985).

11. $\mathrm{M}^{\mathrm{a}} \mathrm{A}$. Rodríguez, J. Rubio, F. Rubio, $\mathrm{M}^{\mathrm{a}} \mathrm{J}$. Liso, J.L. Oteo. "Application of inverse gas chromatography to the study of the surface properties of slates", Clays Clay Miner. 45 (1997) 5, 670-680.

12. M ${ }^{\mathrm{a}}$ A. Rodríguez, F. Rubio, J. Rubio, Maj. Liso, J.L. Oteo. “Caracterización estructural y comportamiento térmico de una muestra de pizarra empleada como material para la edificación. Bol. Geo. Miner. 1 (1995) 437-445.

13. F. M. Nelsen, F. T. Eggertsen. "Adsorption measurement by continuous flow method". Anal Chem. 30 (1958) 1937-1940.

14. M.I. Nieto. "Desarrollo de un método cromatográfico para determinar superficies específicas en sólidos vítreos". Tesina Licenciatura. U. Complutense. Madrid. (1974).

15. V.C. Farmer. The infrared spectra of minerals. Ed. Mineral Soc. London. (1974).

16. A. Corma, A. Mifsud, E. Sanz. "Influence of the chemical composition and textural characteristic of palygorskite on the acid leaching of octahedral cations". Clays Clay Miner. 22 (1987) 225-231.

17. A. Corma, A. Mifsud, E. Sanz. "Kinetics of the acid leaching of palygosrkite: Influence of the octahedral sheet composition". Clay Miner. 25 (1990) 197-205.

18. J.D. López-Gonzalez, A. Ramirez, F. Rodríguez-Reinoso, C. Valenzuela, L. Zurita "Activation of sepiolite with $\mathrm{HNO}_{3}$ solutions and heat treatments: I. Study of the specific surface area". Clay Miner. 16 (1981) 103-113.

19. M.A. Vicente, J.D. López-González, M.A. Bañares. "Acid activation of a Spanish sepiolite: Physico-chemical characterization, free silica content and surface area of the obtained products". Clay Miner. 29 (1994) 361-367.

Recibido: 08.01.01

Aceptado: 26.11 .01

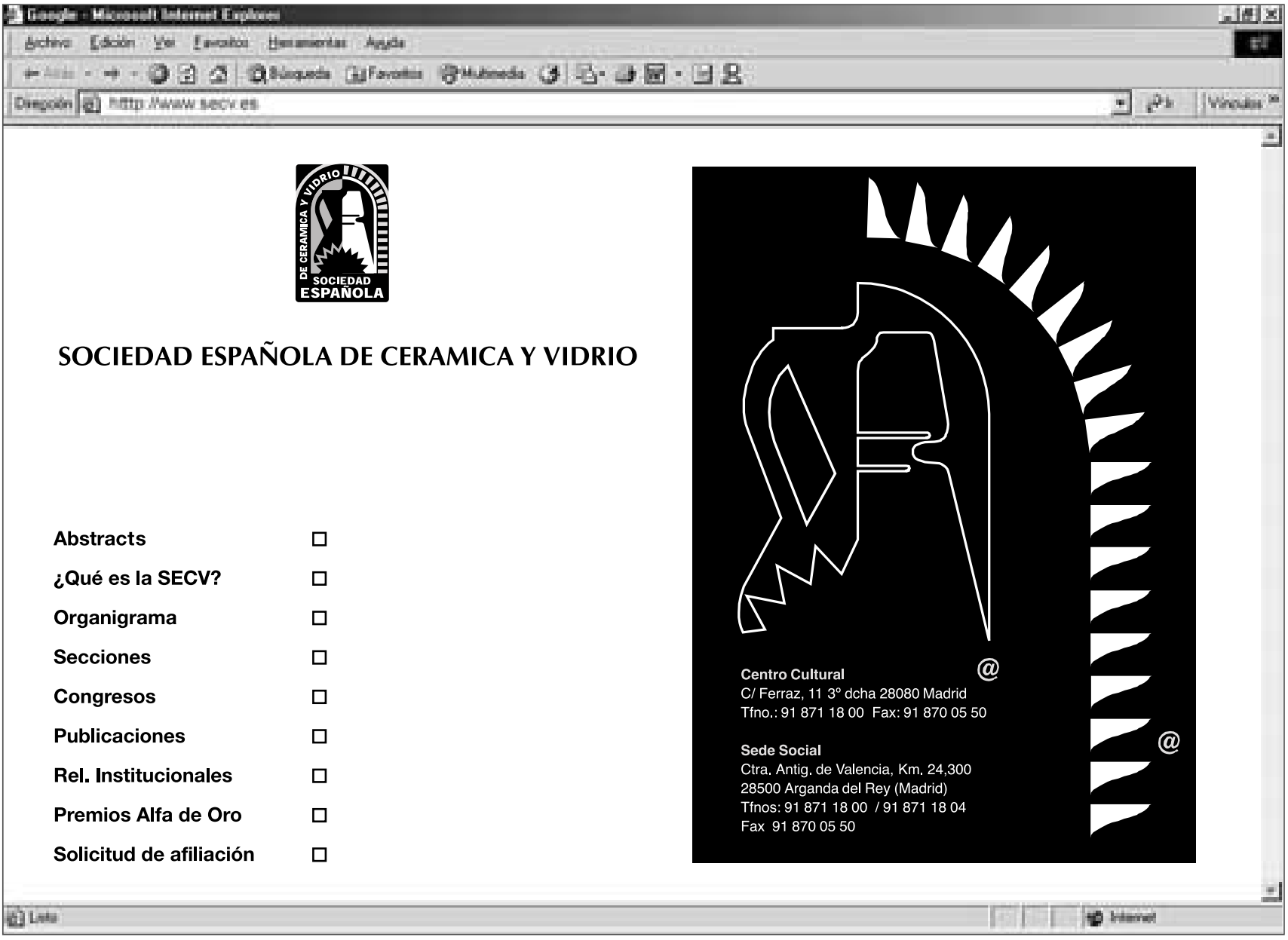

VISITE NUESTRA PÁGINA WEB EN: http://www.secv.es 